\title{
Protection mediated by chemokine CXCL10 in BALB/c mice infected by Leishmania infantum
}

\author{
Webertty Mayk Eufrásio Figueiredo', Sayonara de Melo Viana', Dorotheia Teixeira Alves', \\ Priscila Valera Guerra', Zirlane Castelo Branco Coêlho², Helene Santos Barbosa ${ }^{3}$, Maria Jania Teixeira ${ }^{1 /+}$
}

${ }^{1}$ Universidade Federal do Ceará, Faculdade de Medicina, Departamento de Patologia e Medicina Legal, Fortaleza, CE, Brasil ${ }^{2}$ Universidade Federal do Ceará, Faculdade de Farmácia, Odontologia e Enfermagem, Departamento de Análise Clínica, Fortaleza, CE, Brasil

${ }^{3}$ Fundação Oswaldo Cruz-Fiocruz, Instituto Oswaldo Cruz, Laboratório de Biologia Estrutural, Rio de Janeiro, RJ, Brasil

BACKGROUND Visceral leishmaniasis (VL) caused by Leishmania infantum is characterised by the loss of the ability of the host to generate an effective immune response. Chemokines have a direct involvement in the pathogenesis of leishmaniasis, causing a rapid change in the expression of these molecules during infection by Leishmania.

OBJECTIVES Herein, it was investigated the role of CXCL10 in controlling infection by L. infantum.

METHODS RAW 264.7 macrophages were infected with L. infantum in vitro and treated or not with CXCL10 (25, 50 and 100 ng/ $\mathrm{mL}$ ). Parasite load, as well as nitric oxide (NO), IL-4 and IL-10 production were assessed at 24 and $48 \mathrm{~h}$ after infection. In vivo, $\mathrm{BALB} / \mathrm{c}$ mice were infected and treated or not with CXCL10 $(5 \mu \mathrm{g} / \mathrm{kg})$ at one, three and seven days of infection. Parasite load, IFN- $\gamma$, IL-4, TGF- $\beta$ and IL-10 were evaluated one, seven and 23 days post treatment.

FINDINGS In vitro, CXCL10 reduced parasitic load, not dependent on NO, and inhibited IL-10 and IL-4 secretion. In vivo, CXCL10 was able to reduce the parasite load in both liver and spleen, four weeks after infection, representing a higher decrease in the number of parasites in these organs, also induced IFN- $\gamma$ at day 23 after treatment, correlating with the decrease in parasite load, and reduced IL-10 and TGF- $\beta$.

MAIN CONCLUSIONS This study suggests a partial protective role of CXCL10 against $L$. infantum, mediated by IFN- $\gamma$, not dependent on NO, and with suppression of IL-10 and TGF- $\beta$. These data may provide information for the development of new approaches for future therapeutic interventions for VL.

Key words: L. infantum - CXCL10 - mice - nitric oxide - cytokines - macrophages

Visceral leishmaniasis (VL) is caused by protozoa of the genus Leishmania, in the Americas by L. chagasi (L. infantum synonymy) (Maurício et al. 2000) and in the Old World by L. donovani and L. infantum (Piscopo \& Mallia 2006). These species, known as viscerotropic, preferably infect macrophages and dendritic cells in the bowels, and parasites are promptly found in the liver, spleen and bone marrow (Wilson et al. 2005). The clinical manifestations of the disease can vary from asymptomatic infection to a progressive visceral disease, which if untreated can lead to death and is characterised by fever, weight loss, hypergammaglobulinemia, hepatosplenomegaly, anemia, thrombocytopenia, leukopenia and immunosuppression (Piscopo \& Mallia 2006).

Several experimental models of VL in rodents have been developed, and although none completely reproduced the human disease, the studies using these models have been shown to be very important because they allow investigating the immunological mechanisms and histo-

doi: 10.1590/0074-02760160529

Financial support: CNPq, CAPES, CAPES-Programa DINTER (Doutorado

Interinstitucional) Medicina Tropical 309/2013.

+ Corresponding author: mjteixeira601@gmail.com

Received 12 December 2016

Accepted 4 March 2017 pathological aspects of the disease (Wilson et al. 2005, Nieto et al. 2011). BALB/c mice, when infected by $L$. donovani or $L$. infantum, can resolve the infection spontaneously after some time. In these animals, the effect of IL-12 is delayed for four weeks after infection, at which occurs the appearance of lymphocytes producing IFN- $\gamma$ antigen-specific, granuloma formation in the liver, and development of an antigen-specific response of $\mathrm{CD} 4+$ and CD8+ cells (Wilson et al. 2005). A remarkable feature of the experimental infection of mice with the viscerotropic species of Leishmania is the distinct organ-specific immune response. An acute infection occurs in the liver, which can be resolved between the 4th and 6th week of infection, with subsequent immunity to re-infection, while in the spleen, the parasites may persist (Stanley \& Engwerda 2007). The control of liver disease in mice requires a coordinated response of the host, involving the development of granulomas around the infected macrophages (Murray et al. 2002, Nieto et al. 2011). In contrast, spleen and bone marrow become chronically infected by mechanisms that are not well understood. In the spleen, the persistence of the parasite is accompanied by a failure in the formation of granulomas, splenomegaly, and disruption of lymphoid tissue microarchitecture (Wilson et al. 2005, Stanley \& Engwerda 2007, Nieto et al. 2011).

Several studies have shown that chemokines have a direct involvement in the pathogenesis of leishmaniasis, causing a rapid change in the expression of these 
molecules during infection by Leishmania (Teixeira et al. 2006). In the liver, at the beginning of infection, parasitised Kupffer cells secrete chemokines such as CCL3, CCL2 and CXCL10, which stimulate the recruitment of monocytes and granulocytes (Stanley \& Engwerda 2007). CXCL10 belongs to a large subpopulation of cytokines, which are critical mediators to the function of leukocytes, polarisation of Th1 cells, and activation and trafficking of cells involved in inflammatory responses (Teixeira et al. 2006). CXCL10 binds with high affinity to its CXCR3 receptor (Chen et al. 2004), known to be expressed in many cell types, including CD4+, CD8+, memory cells, NK cells and some subpopulations of dendritic cells (Mohan et al. 2005). Injection of CXCL10 in $\mathrm{BALB} / \mathrm{c}$ mice infected with $L$. major has been shown to induce a strong recruitment and activation of NK cells (Müller et al. 2001). Exogenous CXCL10 is able to reduce the parasitic load of macrophages infected with L. amazonensis in vitro, and in BALB/c mice infected with $L$. amazonensis, can decrease the size of the lesion and parasite load, followed by an increase in IFN- $\gamma$, IL-12 and nitric oxide (NO) (Vásquez \& Soong 2006). In VL, studies in $\mathrm{BALB} / \mathrm{c}$ mice have shown that CXCL10 is involved in the protective response of infection by $L$. donovani, inducing a Th1 response through the regulation of the pathway of inflammatory mediators, such as NO and inflammatory cytokines (Gupta et al. 2009). CXCL10 may participate in the protection against $L$. donovani with remarkable decrease of immunoregulatory cytokines, IL-10 and TGF$\beta$, secreted by T CD4+cells (Gupta et al. 2011).

Herein, it was assessed whether treatment with CXCL10 would confer protection against infection by $L$. infantum in macrophages and in $\mathrm{BALB} / \mathrm{c}$ mice.

\section{MATERIALS AND METHODS}

Animals - A total of $108 \mathrm{BALB} / \mathrm{c}$ mice, male, eight weeks old, obtained from the Biotério Central do Departamento de Patologia e Medicina Legal da Universidade Federal do Ceará, CE, Brazil (DPML/UFC-CE), were used in the experiments. The animals were maintained at $25^{\circ} \mathrm{C}$ with appropriate commercial ration and water ad libitum. The project was approved by Comitê de Ética em Pesquisa Animal (CEPA) at the UFC, under registration number 52/2014.

Parasites - Promastigotes of L. infantum (MHOM/BR/ BA-262) were maintained in a golden hamster. After being recovered from the animal, the parasites were cultured at $25^{\circ} \mathrm{C}$ in N.N.N. medium, containing Schneider's insect medium (Sigma-Aldrich) supplemented with 20\% fetal bovine serum (Sigma-Aldrich), 2\% sterile human urine and antibiotics (100 U/mL penicillin and $100 \mathrm{mg} / \mathrm{mL}$ streptomycin) (all Sigma-Aldrich). The virulence of the strain was maintained by regular passage in a golden hamster. For infection, the parasites were used until the 5th passage, in vitro.

In vitro assays with macrophages - RAW 264.7 macrophages culture were centrifuged at $400 \mathrm{x} g$ for $15 \mathrm{~min}$ at $5^{\circ} \mathrm{C}$, and the cell suspension was adjusted in RPMI 1640 medium supplemented with $10 \mathrm{mM}$ HEPES, 100 $\mathrm{U} / \mathrm{mL}$ penicillin, $100 \mu \mathrm{g} / \mathrm{mL}$ streptomycin, and $2 \mathrm{mM}$ L-glutamine (all from Sigma-Aldrich). Then, the cells were distributed in 24-well plates, each well containing a round glass coverslip of $23 \mathrm{~mm}$ at a concentration of $1 \times 10^{6}$ cells/coverslip. Macrophages were incubated for 24 $\mathrm{h}$ with $5 \% \mathrm{CO}_{2}$ at $37^{\circ} \mathrm{C}$ and $95 \%$ humidity. Cells not adhered were removed by washing with warm RPMI $\left(37^{\circ} \mathrm{C}\right.$; three times) and cultured with RPMI supplemented with or without $L$. infantum live promastigotes, a ratio of 10:1 cell parasites for $12 \mathrm{~h}$. Extracellular parasites were removed by washing (three times) with warm RPMI $\left(37^{\circ} \mathrm{C}\right)$. After this period, the cells were treated with $16 \mathrm{mg} / \mathrm{mL}$ of pentavalent antimony (Glucantime), CXCL10 (25, 50 and $100 \mathrm{ng} / \mathrm{mL}$ ) and LPS (20 ng/mL; positive control) and then were cultured for 24 and $48 \mathrm{~h}$ at $37^{\circ} \mathrm{C}, 5 \% \mathrm{CO}_{2}$ and $95 \%$ humidity. Culture supernatants were collected at 24 and $48 \mathrm{~h}$ to the dosage of cytokines and NO.

Determination of macrophage infection - To quantify the level of infection of macrophages, coverslips containing the cells were washed with saline, and then fixed and stained with Giemsa (Sigma-Aldrich). Stained coverslips were mounted on slides and examined under optical microscopy at a magnification of $100 \mathrm{X}$. The slides were examined to determine the number of amastigotes/50 macrophages.

NO assay - Macrophages culture supernatants were tested for NO release in the form of nitrite $\left(\mathrm{NO}_{2}\right)$ by the Griess reaction. The supernatants were incubated with freshly prepared Griess reagent $(1: 1 \mathrm{v} / \mathrm{v})$ for $10 \mathrm{~min}$ at room temperature. The absorbance was measured spectrophotometrically at $540 \mathrm{~nm}$ and the $\mathrm{NO}_{2}$ concentration determined using a standard curve of sodium nitrite and expressed as $\mu \mathrm{mol} / \mathrm{mL}$. To avoid interference from $\mathrm{NO}_{2}$ possibly present in the medium, a white test was performed for each experiment.

Infection and treatment with CXCL10 in BALB/c mice - Metacyclic promastigotes at the stationary phase were inoculated intraperitoneally into BALB/c mice $(n=36)$ at a concentration of $2 \times 10^{7}$ parasites in $20 \mu \mathrm{L}$ of saline. The animals were divided into two groups, with 18 animals each. One group received CXCL10 $(5 \mu \mathrm{g} / \mathrm{kg})$ (Thermo Scientific) and the other received saline (Untreated group) intraperitoneally after 1, 3 and 7 days of infection (Gupta et al. 2009). The animals were euthanised after 1, 7 and 23 days of treatment by inhalation of Halothane (SigmaAldrich). Six animals per group were euthanised on each period. The animals were weighed before starting treatment and every euthanasia to achieve a weight analysis.

Determination of parasite numbers in liver and spleen - The number of parasites in the liver and spleen was quantified using the technique of limiting dilution as previously described (Titus et al. 1985). Briefly, the animals were euthanised and submerged in 3\% iodised alcohol up to $3 \mathrm{~min}$ to allow decontamination. Then the spleen and liver were removed aseptically, weighed, and one fragment from each organ (spleen: from 0.067 to $0.070 \mathrm{~g}$; liver: from 0.26 to $0.30 \mathrm{~g}$ ) was homogenised in $1 \mathrm{~mL}$ of Schneider medium and left to rest for $5 \mathrm{~min}$. Eight dilutions were made from this cell suspension $\left(10,50,100,500,10^{3}, 10^{4}, 10^{5}\right.$ and $\left.10^{6}\right)$ in Schneider's medium supplemented with $20 \% \mathrm{FBS}, 2 \%$ sterile human urine, and antibiotics $(100 \mathrm{U} / \mathrm{mL}$ penicillin and 100 
$\mu \mathrm{g} / \mathrm{mL}$ streptomycin) (All from Sigma-Aldrich). One hundred microliters of these dilutions were distributed into 96-well plates, flat bottom, and six wells/dilution. The plates were sealed and incubated at $25^{\circ} \mathrm{C}$ for three weeks. The wells were observed on an inverted optical microscope (Nikon) every 3 days to record the dilutions containing promastigotes. The final number of parasites was determined using the ELIDA 12c software.

Culture of splenocytes - Spleen cells were isolated from animals infected with $L$. infantum promastigotes, treated or untreated with CXCL10 (six animals per group) and separated by centrifugation with Ficoll, $800 \mathrm{x} g$ for $30 \mathrm{~min}$ at $25^{\circ} \mathrm{C}$, after one, seven and 23 days after treatment. The cells were washed with RPMI $400 \times \mathrm{g}$ for 15 min at $5^{\circ} \mathrm{C}$ and then cultured in 96-well plates $\left(1 \times 10^{6}\right.$ cells $/ \mathrm{mL}$ ) in RPMI medium supplemented with $10 \%$ fetal bovine serum (FBS), $50 \mu \mathrm{M}$ 2-mercaptoethanol, $2 \mathrm{mM} \mathrm{L-}$ glutamine and antibiotics $(100 \mathrm{U} / \mathrm{mL}$ penicillin, $100 \mathrm{mg} /$ $\mathrm{mL}$ streptomycin) with or without live promastigotes at a concentration of $1 \times 10^{7} / \mathrm{mL}$, at $37^{\circ} \mathrm{C}, 5 \% \mathrm{CO}_{2}$. As control, cells were isolated for the spleen of non-infected BALB/c mice. The supernatants were collected after $48 \mathrm{~h}$ and preserved at $-20^{\circ} \mathrm{C}$ for subsequent dosing of cytokines.

Cytokines assay - Levels of cytokines obtained from both the culture of macrophages (only IL-4 and IL10 ) and culture of splenocytes (IFN- $\gamma$, IL- 4, IL-10 and TGF- $\beta$ ) were determined using the ELISA technique as recommended by the kit manufacturer (BD Biosciences). The results were analysed using the Softmax PRO software (Molecular Devices).

Statistical analysis - $t$-Student test was applied to verify the statistical significance between the treated and the untreated group. For comparisons between multiple groups it was used the one-way ANOVA test, followed by Bonferroni post-test. The tests were performed using GraphPad Prism Software version 5.00 (GraphPad Software, San Diego, CA, USA). The results are presented as arithmetic mean and standard error of the mean. In all tests, the minimum significance was accepted when $\mathrm{p}<0.05$.

\section{RESULTS}

Parasite load in macrophages - Treatment with CXCL10 resulted in a significant reduction of the parasitic load at concentrations of $50 \mathrm{ng} / \mathrm{mL}(70.3 \%)$ and 100 $\mathrm{ng} / \mathrm{mL}(74.0 \%)$ compared to Glucantime $(50.4 \%)$ and untreated animals, after $24 \mathrm{~h}$ (Fig. 1A). After 48 h, treatment with CXCL10 maintained reduction in the number of intracellular parasites at concentrations of $50 \mathrm{ng} / \mathrm{mL}$ (70\%) and $100 \mathrm{ng} / \mathrm{mL}(72.6 \%)$ when compared to Glucantime (61.8\%) and untreated (Fig. 1B).

NO production by macrophages - After $24 \mathrm{~h}, \mathrm{NO}$ production behaved in a dose-dependent manner in samples that were treated with CXCL10, with a production average of from $79 \mu \mathrm{mol} / \mathrm{L}$ to $282 \mu \mathrm{mol} / \mathrm{L}$ (Fig. 2A). After $48 \mathrm{~h}$, there was a reduction of NO production, CXCL10 induced average production ranging from $52 \mu \mathrm{mol} / \mathrm{L}$ to $164 \mu \mathrm{mol} / \mathrm{L}$; still showing a dose-dependence (Fig. 2B).
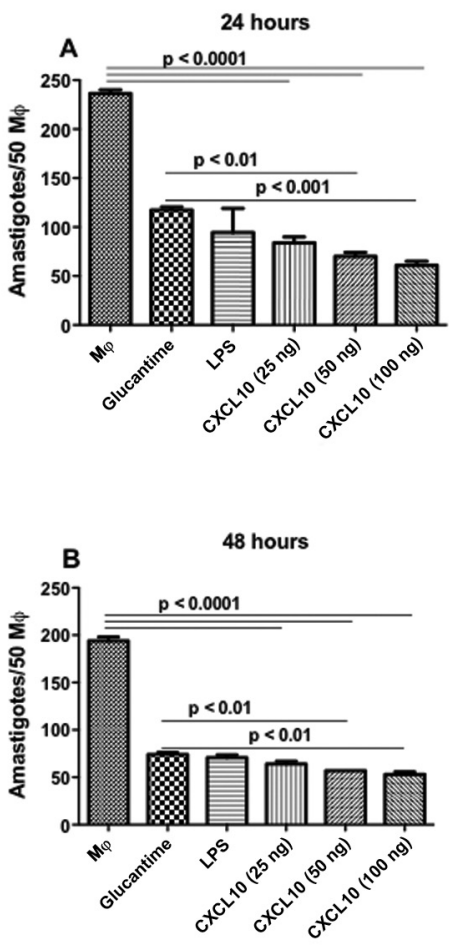

Fig. 1: number of parasites in macrophages infected with Leishmania infantum and treated or untreated with CXCL10. RAW 264.7 macrophages were infected with promastigotes of $L$. infantum (10 parasites: 1 macrophage) and after $12 \mathrm{~h}$ of infection the cells were treated in vitro with CXCL10 (25, 50 and $100 \mathrm{ng} / \mathrm{mL})$, LPS $(20 \mathrm{ng} / \mathrm{mL})$ and Glucantime (16 mg/mL). After incubation 24 (A) and $48 \mathrm{~h}(\mathrm{~B})$, the number of amastigotes per 50 macrophages was determined under an optical microscope. Results are expressed as mean \pm standard error of mean, and are representative of three independent experiments.

Cytokine production in vitro - Treatment with CXCL10 (100 ng/mL) in macrophages infected by $L$. infantum inhibited IL-4 production (significant at $24 \mathrm{~h}$ post-infection; $p<0.01)$ and IL-10 $(p<0.001)$ in two time periods (Fig. 3A-B). At $48 \mathrm{~h}$ after infection, the results were similar for both IL-4 and IL-10 (Fig. 3A-B).

Relative weight of the liver and spleen - The analysis of the relative weight of the spleen and liver was measured one, seven, 23 and 45 days after treatment. It was observed that on the 1st day after treatment, the treated and the untreated group showed no differences in the weight of the spleen, although the weight of the spleen of both groups (untreated: $0.0056 \pm 0.003$; treated: $0.0045 \pm 0.0004$ ) was slightly higher than those found in healthy animals $(0.0042$ \pm 0.0002 ) (Fig. 4A). There was a decrease in spleen weight of the treated animals after the 7th day, unlike what happened with the untreated group, whose weights only decreased after 45 days of treatment, and yet were above the weight of the spleen of healthy animals. When the groups were compared, it was observed that there were differences between them on 7th day $(p=0.0286)$ and 23 after treatment $(\mathrm{p}=0.0033)$ (Fig. 4A). Regarding the liver, it was observed that the weight of this organ decreased after the 7 th day of treatment in treated animals, when compared 

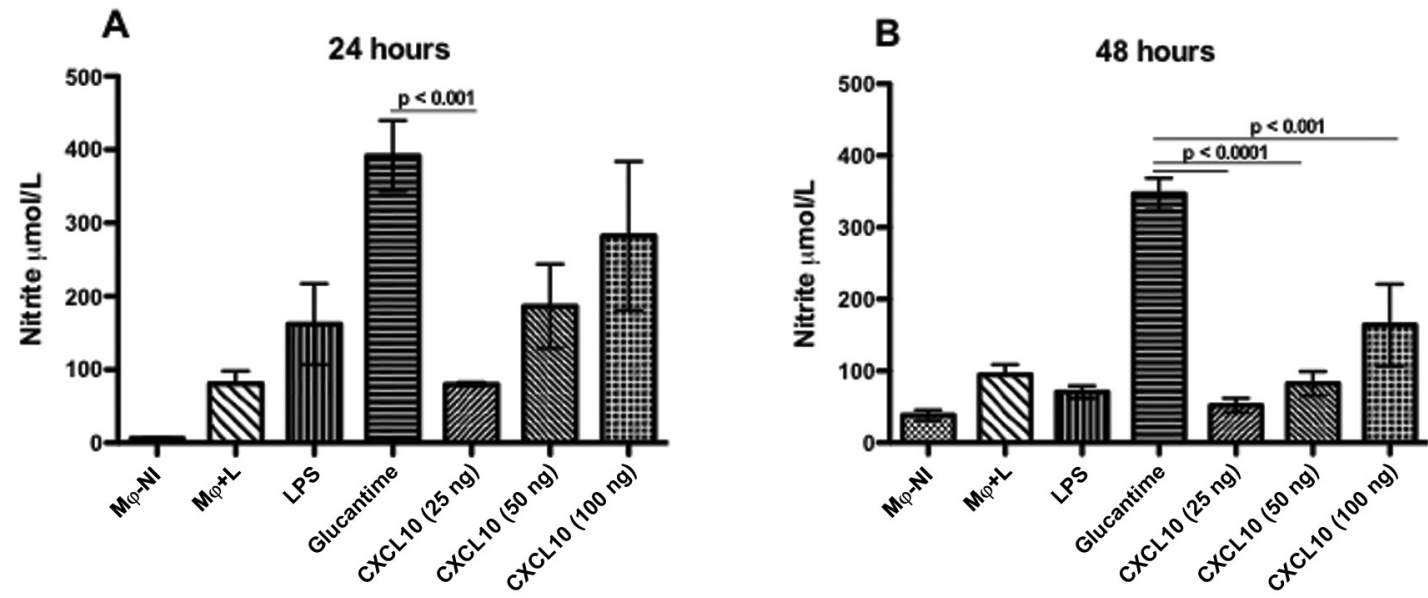

Fig. 2: nitric oxide (NO) by macrophages infected with Leishmania infantum, and treated or untreated with CXCL10. RAW 264.7 macrophages were infected and treated as described in Fig. 1. Culture supernatants were collected with $24 \mathrm{~h} \mathrm{(A)}$ and $48 \mathrm{~h}$ (B) for the measurement of NO in the form of nitrite $\left(\mathrm{NO}_{2}\right)$. The data represent the arithmetic mean \pm standard error of the mean, and are representative of three independent experiments. $\mathrm{M} \varnothing+\mathrm{NI}=$ macrophage not infected; $\mathrm{M} \varnothing+\mathrm{L}=$ macrophage infected with $L$. infantum.
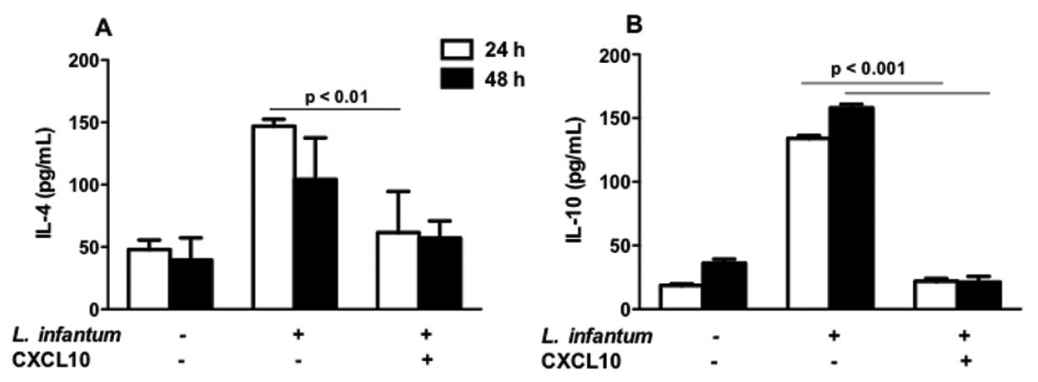

Fig. 3: production of IL-4 and IL-10 by macrophages infected with Leishmania infantum and treated or untreated with CXCL10. RAW 264.7 macrophages were infected with $L$. infantum and, after $12 \mathrm{~h}$, treated in vitro with CXCL10 $(100 \mathrm{ng} / \mathrm{mL})$. Culture supernatants were collected with $24 \mathrm{~h}$ (A) and $48 \mathrm{~h}$ (B) for the measurement of cytokines. Results are expressed as mean \pm standard error of mean, and are representative of three independent experiments.

to untreated animals. Nevertheless, no difference was observed between the groups, although the liver weight in untreated animals showed a slight tendency to increase more than the treated animals, when compared with the liver weight of healthy animals (Fig. 4B).

Number of parasites in the spleen and liver - The results showed that after the 1st day of treatment, animals treated with CXCL10 showed no difference in the number of parasites in the spleen and liver when compared to untreated animals (Fig. 5A-B). As expected, the liver was the most infected organ in the early eight days of infection (means: one day after treatment), presenting in both groups (treated and untreated) a number of parasites near the $10^{6}$ (Fig. 5B). On the other hand, it was found in the spleen approximately half of the initial inoculum, about $10^{4}$ parasites in both groups (treated and untreated) (Fig. 5A). After seven days of treatment, the number of parasites in treated animals remained stable in both the spleen (approximately $10^{4}$ ) and the liver (approximately $10^{6}$ ) (Fig. 5A-B). After 23 days of treatment, there was a decline in the number of parasites in target organs in both the treated and untreated groups. However, this decrease in parasite load was found to be significant only in the spleen of the treated group $(p=0.0272)$, when compared to the untreated group (Fig. 5A). Even at that time, it was observed that in the treated group, in both organs, the number of parasites reached the lowest values (approximately $10^{2}$ in spleen and $10^{3}$ in the liver) (Fig. $5 \mathrm{~A}-\mathrm{B})$. The group that received CXCL10 in different time periods showed that in the spleen there was a difference $(\mathrm{p}<0.05)$ between 1-23 days post treatment (Fig. 5A). In the liver, there was also a significant decrease $(\mathrm{p}<0.05)$ from day seven to day 23 post treatment in the treated group, showing a reduction in parasite load equivalent to that found in the spleen. Although there was an apparently similar reduction in liver rate between treated and untreated groups from day seven to day 23 post treatment, in fact, there was no statistical significance (Fig. 5B).

Production of cytokine in vivo - IFN- $\gamma$ was produced in a more outstanding way by splenocytes from treated animals than the untreated animals, in the three time periods evaluated (after one day, $\mathrm{p}=0.0218$; seven days, $\mathrm{p}$ 

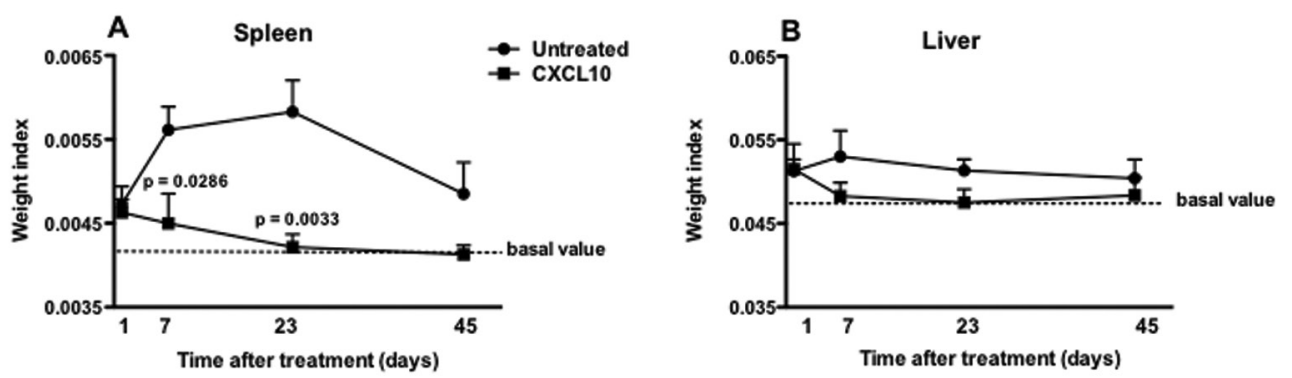

Fig. 4: relative weight of spleen (A) and liver (B) of BALB/c mice infected with Leishmania infantum and treated or not with CXCL10. The animals were treated with CXCL10, intraperitoneally ( $5 \mu \mathrm{g} / \mathrm{kg}$ ), or saline at one, three and seven days of infection. At one, seven, 23 and 45 days after the treatment the animals were euthanised and the spleen and liver were removed. Data from six animals per group are represented by arithmetic mean \pm standard error. The dashed lines represent the arithmetic mean of the relative weight of spleen $(0.0042)$ and liver $(0.048)$ of six healthy animals (basal value).

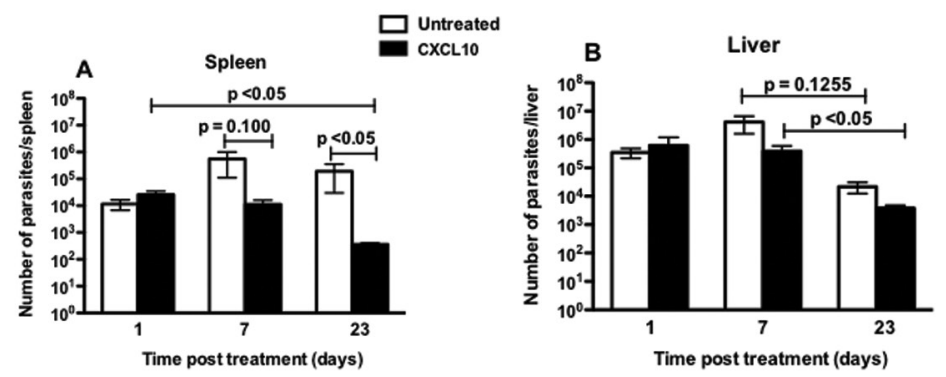

Fig. 5: parasite load in the spleen (A) and liver (B) of BALB/c mice infected with Leishmania infantum and treated or untreated with CXCL10. Animals were treated as described in Fig. 4. After one, seven and 23 days of treatment, animals were euthanised; the spleen and liver were removed and used for evaluating the parasite load. Data from six animals per group are represented by the arithmetic mean \pm standard error of the mean, and are representative of three independent experiments.
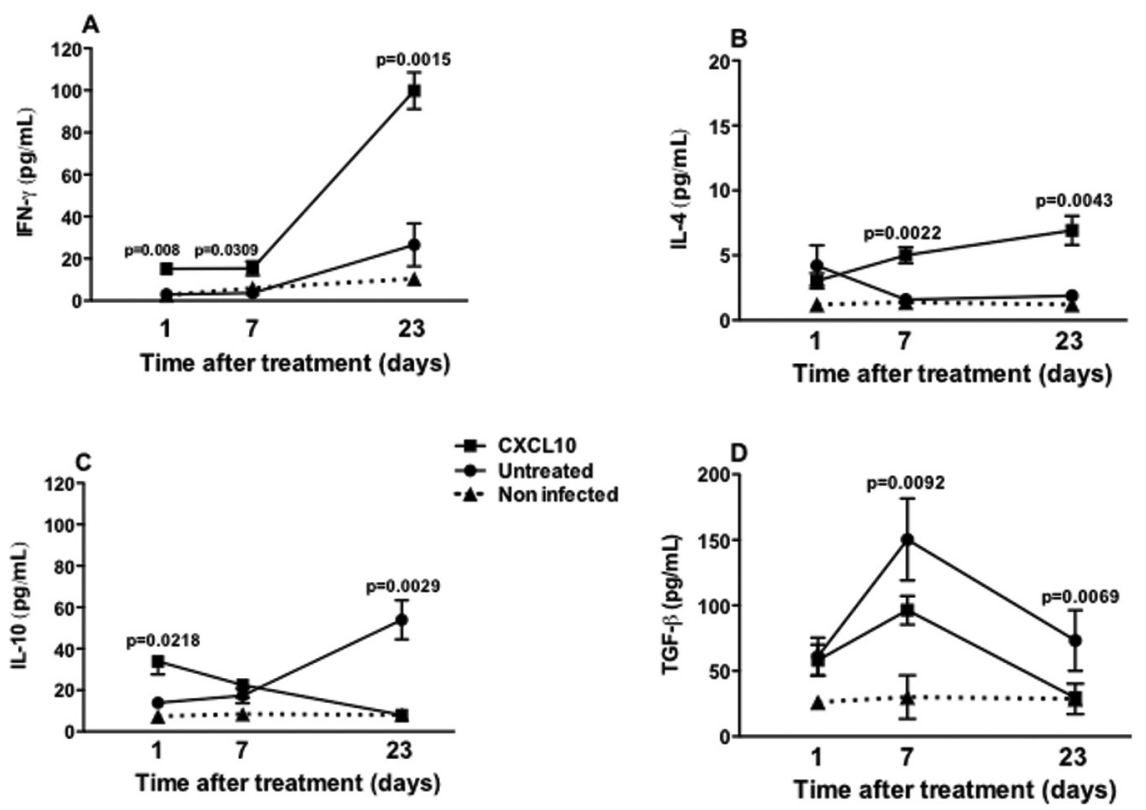

Fig. 6: production of IFN- $\gamma$ (A), IL-4 (B), IL-10 (C) and TGF- $\beta$ (D) by splenocytes from BALB/c mice infected with Leishmania infantum and treated $(\boldsymbol{\bullet})$ untreated $(\bullet)$ with CXCL10, or non-infected (NI) ( $(\boldsymbol{\Delta})$. Animals were treated as described in Fig. 4. After one, seven and 23 days of treatment, the animals were euthanised to obtain the splenocytes. The supernatants of these cells were collected at $48 \mathrm{~h}$ for the measurement of the cytokines. Data from six animals per group are shown as the arithmetic mean \pm standard error of the mean, and are representative of three independent experiments. 
$=0.0309$; and after 23 days of treatment, $p=0.0015)$. In animals treated with CXCL10, IFN- $\gamma$ production reached its maximum value of $100 \mathrm{pg} / \mathrm{mL}$ at day 23 post treatment, which was equivalent to a five-fold increase relative to the initial concentration of $20 \mathrm{pg} / \mathrm{mL}(\mathrm{p}<0.05)$ (Fig. 6A). In both groups, IL-4 production remained always below of $10 \mathrm{pg} / \mathrm{mL}$, at all three time periods evaluated (Fig. 6B). After the 1st day of treatment there was no difference between groups, however, in the last two days of the study it was observed that animals treated with CXCL10 induced a higher production of IL-4, compared with those untreated (seven days post treatment, $\mathrm{p}$ $=0.0022 ; 23$ days post treatment, $\mathrm{p}=0.0043$ ) (Fig. 6B). In the animals treated with CXCL10, IL-10 production was twice as high as that of IFN- $\gamma$ at one and 23 days after treatment, reaching a concentration of approximately $40 \mathrm{pg} / \mathrm{mL}$, and also significantly higher than that of untreated animals $(p=0.0218)$ in the same time period (Fig. $6 \mathrm{C})$. After seven days of treatment, there was no difference in IL-10 between CXCL10-treated and untreated animals, however, after 23 days, it fell significantly in the treated animals $(p=0.0029)$ (Fig. 6C). By contrast, in the untreated group, the production of IL-10 remained growing, with about $60 \mathrm{pg} / \mathrm{mL}$ being detected after 23 days of treatment (Fig. 6C). Regarding TGF- $\beta$, it was observed that the levels of this cytokine were always much higher when compared with the other cytokines evaluated. After the 1st day of treatment, both the untreated and the treated group had similar levels of TGF- $\beta$, about 50 $\mathrm{pg} / \mathrm{mL}$ (Fig. 6D). After seven days of treatment, the production of TGF- $\beta$ was lower in the treated group, about $100 \mathrm{pg} / \mathrm{mL}$, lower than that produced by the untreated group ( $150 \mathrm{pg} / \mathrm{mL} ; \mathrm{p}=0.0092)$ (Fig. 6D). After 23 days of treatment, the production of this cytokine decreased in both groups (treated and untreated) (Fig. 6D).

\section{DISCUSSION}

This study evaluated the potential of CXCL10 in controlling infection caused by L. infantum using in vitro and in vivo models of VL. In vitro, CXCL10 (100 ng/ $\mathrm{mL}$ ) was able to reduce by $74.0 \%$ the intracellular parasite burden compared to infected and untreated macrophages. Also, in vivo, it was observed that treatment with CXCL10 was able to reduce the parasite load in both liver and spleen, four weeks after infection, representing a higher decrease in the number of parasites in these organs. This decrease in the number of parasites in the spleen correlated with the decrease in size of this organ in treated animals compared to untreated animals. During infection by $L$. infantum in mice, the amastigotes multiply rapidly during the first two weeks in the liver and disappear spontaneously around the 8th week of infection, whereas in the spleen parasites grow more slowly, leading to a chronic infection in this organ (Nieto et al. 2011). The results of parasite load found in this study corroborate the findings of the literature and more importantly, suggest a protective role of CXCL10 in infection by $L$. infantum, since this chemokine was able to induce a augmented reduction in the number of parasites in the two target organs of the treated animals, which was not observed in the untreated group.
The probable effector mechanism of this parasitic reduction is related to macrophage activation induced by CXCL10, since it has been reported that some chemokines such as CCL2 and CXCL10, can activate macrophages to participate in reducing the number of parasites (Ritter \& Moll 2000). Macrophages mediate Leishmania destruction mainly via reactive oxygen derivatives and NO production (Vouldoukis et al. 1997). Also, it has been showed that a phenotype of $L$. infantum was resistant to death by apoptosis mediated by NO (Holzmüller et al. 2005). In this present work, the reduction of parasites in vitro do not seem to depend exclusively of $\mathrm{NO}$, since NO production decreased significantly in infected macrophages treated with CXCL10 when compared to those infected and not treated with the chemokine. Some species of Leishmania are resistant to the microbicidal action of NO, as some isolates of L. braziliensis, which exhibited higher intracellular growth even in the presence of LPS and IFN- $\gamma$ (Souza et al. 2010). There are reports that other oxygen metabolism products are involved in the respiratory burst in activated murine macrophages, such as hydrogen peroxide $\left(\mathrm{H}_{2} \mathrm{O}_{2}\right)$ and superoxide anion $\left(\mathrm{O}_{2}\right)$, and thus would also be involved in the elimination of Leishmania (Van Assche et al. 2011) which corroborates the hypothesis suggested in the present work.

In murine models of $\mathrm{VL}$, using BALB/c mice, the control of the disease depends on the magnitude of the Th1 cell response, leading to the production of IFN- $\gamma$, the activation of macrophages (Kaye et al. 2004), the formation of mature hepatic granulomas as well as the production of reactive nitrogen species and oxygen intermediates, which are essential for the elimination of parasites within the Kupffer cells and dendritic cells (Murray 2001, Stanley \& Engwerda 2007). The onset of the disease is associated with a decrease in the response to these factors, which helps the parasite to live and reproduce in the macrophage phagolysosome (Melby et al. 2001). It was found that IFN- $\gamma$ production in the group treated with CXCL10 was augmented when compared to the untreated group in the spleen. This production was significantly increased five times than the concentration produced after the first day of treatment, an increase that coincided with the decrease in parasite load after 23 days post treatment, strengthening the hypothesis of the protective role of CXCL10. Previous studies have demonstrated the importance of IFN- $\gamma$ in the control of visceral infection. Monocytes of healthy individuals previously treated with IFN- $\gamma$ produce, after infection with $L$. donovani, a greater amount of TNF- $\alpha$, important cytokine in the host response to microbial infection (Reiner et al. 1990). Corroborating the high levels of IFN- $\gamma$ and the decrease of the parasite load, it was observed that CXCL10 induced a significant reduction in the production of IL-10 and TGF- $\beta$, regulatory cytokines, suggesting that CXCL10 induced a important Th1 response in BALB/c mice infected with $L$. infantum.

The treatment with CXCL10 in vitro induced a significant decrease of IL-10, anti-inflammatory cytokine and present in high levels during the active stage of the disease. This date confirms the protective response represented by the reduction of the parasitic load, even with decreased 
production of NO. On the animals receiving CXCL10, the concentration of IL-10 was 10 times lower than the concentration of IFN- $\gamma$, however, despite CXCL10 have induced the reduction of TGF- $\beta$ concentration to close proximity to the concentration of IFN- $\gamma$, the cytokine was still able to promote Th1 polarisation and reduction of parasite load. These data are consistent with other studies showing that IL-10 plays an important role in suppressing the protective immune response in murine VL (Murray et al. 2003). The association of immunosuppression induced by IL-10 in human VL is well established. Patients with active disease present high serum levels of IL-10, as well as increased expression of IL-10 mRNA in lesions. IL-10 is a regulatory cytokine that is induced as a homeostatic response, which protects tissues from collateral damage caused by excessive inflammation (Mege et al. 2006).

Recently, studies have also shown that treatment with CXCL10 results in protection in vivo against $L$. donovani, through significant reduction of the immunosuppressive cytokines, IL-10 and TGF- $\beta$, due to modulation of regulatory $T$ cells (Gupta et al. 2011). In the present study, the levels of TGF- $\beta$ in the animals treated with CXCL10 were always much higher than those of other evaluated cytokines. These results does not fully corroborated other studies suggesting that the role of TGF- $\beta$ in the control of parasite load and the resistance of the host, dependent of IFN- $\gamma$, during infection by viscerotropic strains such as L. donovani, seems to be less important compared to IL10 (Murray et al. 2005). Studies with animals deficient in IL-10 and TGF- $\beta$, infected with $L$. infantum and treated with CXCL10 could perhaps clarify this issue.

Unlike the models of murine cutaneous leishmaniasis, in which IL-4 is associated with the Th2 response, in the model of infection by $L$. donovani there are studies showing that IL-4 does not exacerbate the disease and may in fact act in combating the parasite, which is rather paradoxical (Mohrs et al. 1999). These studies showed that IL-4 could act positively regulating immunity against $L$. donovani by increasing the number of mature granulomas and consequent reduction of parasite load in the liver (Stäger et al. 2003). Herein, the concentration of IL-4 in both groups always remained very low at all three time periods analysed. However, in treated animals it was observed greater production of IL-4 compared with the untreated group, concomitant to the earlier presence of mature granulomas in the livers of animals that received the chemokine (data not shown).

More recently, Murray et al. (2016), in experiment performed in the $L$. donovani-infected livers of gene-deficient C57BL/6 mice (CXCL10-/- and CXCR3-/) showed that CXCL10 may promote anticipated granuloma assembly, however, may hinder early parasite control and may has no role in conversion of developing granulomas to histologically mature-appearing structures. Therefore, CXCL10 appears to paradoxically promote liver infection too.

Chemokines may act as promising new therapeutic agents to combat $L$. donovani because, among the types of cell-mediated response, some may induce polarisation of cell response of the Th1 type, able to fight infection (Gupta et al. 2009). Collectively, data from this study suggest an important protective role of CXCL10 in BALB/c mice infected with $L$. infantum mediated by a significant production of IFN- $\gamma$ and suppression of immunoregulatory cytokines, IL-10 and TGF- $\beta$, leading to the hypothesis if it is not associated with a decrease in the frequency of regulatory $\mathrm{T}$ cells.

The beginning of an appropriate immune response is a challenge to control Leishmania infection. Recent approaches to the combination therapy, targeted delivery and use of immunological adjuvants are efforts to reduce the effective dosages of drugs available on the market associated with toxicity (Singh \& Sundar 2014). The data from this study open perspectives for the development of new alternative therapeutic formulations for cutaneous leishmaniasis.

\section{AUTHORS' CONTRIBUTION}

WMEF - Planning and standardisation of assays, done in vitro and in vivo experiments, co-orientation of students, discussion and interpretation of results and paper write; SMV - done in vitro assays with macrophages and splenocytes cultures, dosing nitric oxide (NO) and cytokines from in vitro experiments, coorientation of students, discussion and interpretation of results; DTA - done maintenance and growth curves of Leishmania cultures, in vitro assays with macrophages and splenocytes cultures, and discussion and interpretation of results; PVG - done infection, treatment and follow-up of the mice, dosing cytokines from in vivo experiments, co-orientation of students, discussion and interpretation of results; ZCBC - dosing cytokines from in vivo experiments, co-orientation of students, discussion and interpretation of results; HSB - co-orientation of students, discussion and interpretation of results and paper write; MJT coordinator of work, planning assays, orientation of students, discussion and interpretation of results and paper write.

\section{REFERENCES}

Chen J, Vistica BP, Takase H, Ham DI, Fariss RN, Wawrousek EF, et al. A unique pattern of up- and downregulation of chemokine receptor CXCR3 on inflammation-inducing Th1 cells. Eur J Immunol. 2004; 34(10): 2885-94.

Gupta G, Bhattacharjee S, Bhattacharyya S, Bhattacharya P, Adhikari A, Mukherjee A, et al. CXC chemokine-mediated protection against visceral leishmaniasis: involvement of the proinflammatory response. J Infect Dis. 2009; 200(8): 1300-10.

Gupta G, Majumdar S, Adhikari A, Bhattacharya P, Mukherjee AK, Majumdar SB, et al. Treatment with IP-10 induces host-protective immune response by regulating the $\mathrm{T}$ regulatory cell functioning in Leishmania donovani-infected mice. Med Microbiol Immunol. 2011; 200(4): 241-53.

Holzmüller P, Cavaleyra M, Moreaux J, Kovacic R, Vincendeau P, Papierok G, et al. Lymphocytes of dogs immunized with purified excreted-secreted antigens of Leishmania infantum co-incubated with Leishmania infected macrophages produce IFN-gamma resulting in nitric oxide-mediated amastigotes apoptosis. Vet Immunol Immunopathol. 2005; 106(3-4): 247-57.

Kaye PM, Svensson M, Ato M, Maroof A, Polley R, Stager S, et al. The immunopathology of experimental visceral leishmaniasis. Immunol Rev. 2004; 201: 239-53.

Maurício IL, Stothard JR, Miles MA. The strange case of Leishmania chagasi. Parasitol Today. 2000; 16(5): 188-9.

Mege JL, Meghari S, Honstettre A, Capo C, Raoult D. The two faces of interleukin 10 in human infectious diseases. Lancet Infect Dis. 2006; 6(9): 557-69. 
Melby PC, Chandrasekar B, Zhao W, Coe JE. The hamster as a model of human visceral leishmaniasis: progressive disease and impaired generation of nitric oxide in the face of a prominent Th1like cytokine response. J Immunol. 2001; 166(3): 1912-20.

Mohan K, Cordeiro E, Vaci M, Mcmaster C, Issekutz TB. CXCR3 is required for migration to dermal inflammation by normal and in vivo activated T cells: differential requirements by CD4 and CD8 memory subsets. Eur J Immunol. 2005; 35(6): 1702-11.

Mohrs M, Ledermann B, Köhler G, Dorfmüller A, Gessner A, Brombacher F. Differences between IL-4- and IL-4 receptor alphadeficient mice in chronic leishmaniasis reveal a protective role for IL-13 receptor signaling. J Immunol. 1999; 162(12): 7302-8.

Müller K, van Zandbergen G, Hansen B, Laufs H, Jahnke N, Solbach $\mathrm{W}$, et al. Chemokines, natural killer cells and granulocytes in the early course of Leishmania major infection in mice. Med Microbiol Immunol. 2001; 190(1-2): 73-6.

Murray HW, Berman JD, Davies CR, Saraiva NG. Advances in leishmaniasis. Lancet. 2005; 366(9496): 1561-77.

Murray HW, Lu CM, Mauze S, Freeman S, Moreira AL, Kaplan G, et al. Interleukin-10 (IL-10) in experimental visceral leishmaniasis and IL-10 receptor blockade as immunotherapy. Infect Immun. 2002; 70(11): 6284-93.

Murray HW, Luster AD, Zheng H, Ma X. Gamma interferon-regulated chemokines in Leishmania donovani infection in the liver. Infect Immun. 2016; 85(1): e00824-16.

Murray HW, Moreira AL, Lu CM, Devecchio JL, Matsuhashi M, $\mathrm{Ma} \mathrm{X}$, et al. Determinants of response to interleukin-10 receptor blockade immunotherapy in experimental visceral leishmaniasis. J Infect Dis. 2003; 188(3): 458-64.

Murray HW. Tissue granuloma structure-function in experimental visceral leishmaniasis. Int J Exp Pathol. 2001; 82(5): 249-67.

Nieto A, Domínguez-Bernal G, Orden JA, de la Fuente R, MadridElena N, Carrión J. Mechanisms of resistance and susceptibility to experimental visceral leishmaniosis: BALB/c mouse versus syrian hamster model. Vet Res. 2011; 42: 39.

Piscopo TV, Mallia AC. Leishmaniasis. Postgrad Med J. 2006; 82(972): 649-57.

Reiner NE, Ng W, Wilson CB, McMaster WR, Burchett SK. Modulation of in vitro monocyte cytokine responses to Leishmania donovani. Interferon-gamma prevents parasite-induced inhibition of interleukin 1 production and primes monocytes to respond to Leishmania by producing both tumor necrosis factor-alpha and interleukin 1. J Clin Invest. 1990; 85(6): 1914-24.

Ritter U, Moll H. Monocyte chemotactic protein-1 stimulates the killing of Leishmania major by human monocytes, acts synergistically with IFN-gamma and is antagonized by IL-4. Eur J Immunol. 2000; 30(11): 3111-20.

Singh OP, Sundar S. Immunotherapy and targeted therapies in treatment of visceral leishmaniasis: current status and future prospects. Front Immunol. 2014; 5: 296.

Souza AS, Giudice A, Pereira JM, Guimarães LH, de Jesus AR, de Moura TR, et al. Resistance of Leishmania (Viannia) braziliensis to nitric oxide: correlation with antimony therapy and TNF-alpha production. BMC Infect Dis. 2010; 10: 209.

Stäger S, Alexander J, Carter KC, Brombacher F, Kaye PM. Both interleukin-4 (IL-4) and IL-4 receptor alpha signaling contribute to the development of hepatic granulomas with optimal antileishmanial activity. Infect Immun. 2003; 71(8): 4804-7.

Stanley AC, Engwerda CR. Balancing immunity and pathology in visceral leishmaniasis. Immunol Cell Biol. 2007; 85(2): 138-47.

Teixeira MJ, Teixeira LR, Andrade BB, Barral-Netto M, Barral A. Chemokines in host-parasite interactions in leishmaniasis. Trends Parasitol. 2006; 22(1): 32-40.

Titus RG, Marchand M, Boon T, Louis JA. A limiting dilution assay for quantifying Leishmania major in tissues of infected mice. Parasite Immunol. 1985; 7(5): 545-55.

Van Assche T, Deschacht M, da Luz RA, Maes L, Cos P. Leishmaniamacrophage interactions: insights into the redox biology. Free Radic Biol Med. 2011; 51(2): 337-51.

Vásquez RE, Soong L. CXCL10/gamma interferon-inducible protein 10-mediated protection against Leishmania amazonensis infection in mice. Infect Immun. 2006; 74(12): 6769-77.

Vouldoukis I, Bécherel PA, Riveros-Moreno V, Arock M, da Silva O, Debré $\mathrm{P}$, et al. Interleukin-10 and interleukin-4 inhibit intracellular killing of Leishmania infantum and Leishmania major by human macrophages by decreasing nitric oxide generation. Eur J Immunol. 1997; 27(4): 860-5.

Wilson ME, Jerônimo SM, Pearson RD. Immunopathogenesis of infection with the visceralizing Leishmania species. Microb Pathog. 2005; 38(4): 147-60. 